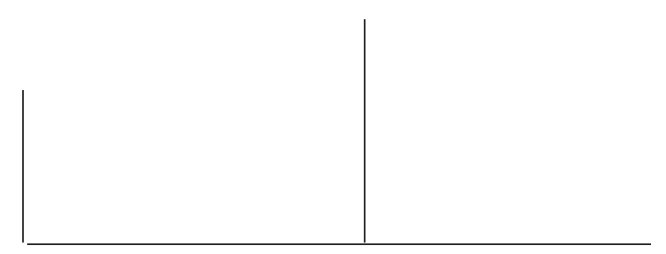

Rev. Latinoam. Psicopat. Fund., V, 3, 44-55

\title{
Filiation et consommation de medicaments psychotropes
}

Alain Ducousso-Lacaze

\begin{abstract}
A partir de deux entretiens semi-directifs l'auteur présente certains aspects des résultats d'une recherche clinique réalisée avec des patients de l'hôpital général consommant des médicaments psychotropes. Il s'agit de mette en évidence comment, pour certains patients, la consommation et la prescription prennent un sens par rapport aux enjeux de la filiation narcissique. Les analyses cliniques portent essentiellement sur la fonction de support imaginaire de la transmission que peuvent revêtir les médicaments ainsi que leur rôle dans les conflits entre appartenance et différenciation à l'égard du groupe familial.
\end{abstract}

Mots clés: Filiation narcissique, médicaments psychotropes,

fantasme de transmission, appartenance, différenciation 
La prescription et la consommation de médicaments psychotropes font aujourd'hui l'objet d'un nombre important de recherches, essentiellement dans le champs de la sociologie et l'anthropologie (Le Moigne, 1999). Elles sont également au centre de réflexions de fond sur les nouvelles formes de l'individualité dans les sociétés occidentales (Ehrenberg, 1995) ainsi que sur l'évolution des pratiques de prise en charge de la souffrance psychique (Zarifian, 1996). Indépendamment des polémiques je retiendrai deux constats. Il apparaît tout d'abord, à partir d'une revue de question de l'ensemble de ces travaux, qu' "au delà des catégories de la biologie, de la chimie et de la médecine, patients et médecins ont fini par imposer leurs propres logiques d'action" (Le Moigne, 1999, p. 9); d'autre part les approches actuelles ne parviennent pas à "étudier la consommation 'de l'intérieur"' du fait qu'elles se centrent tantôt sur des données sociodémographiques, tantôt "sur les faits les plus saillants du changement identitaire (rupture du statut social, décomposition familiale, déclaration de morbidité) sans pouvoir les resituer dans le cadre d'une biographie individuelle" (Ibid., p. 31).

La recherche ${ }^{1}$ dont il sera question ici s'est en revanche donné pour objectif d'étudier l'expérience vécue de personnes consommant des médicaments psychotropes. Centrée sur le discours des patients, dans une perspective clinique référée à la théorie psychanalytique, elle tente d'appréhender la signification subjective de la consommation comme de l'acte de prescription. Dans le présent article je rendrai compte seulement de certains aspects de nos résultats, ceux qui permettent de montrer comment la prescription et la consommation de médicaments psychotropes peuvent solliciter le patient en tant que sujet de la filiation.

1. Recherche initialement financée par le Programme Hospitalier de Recherche Clinique et menée par l'équipe Clinique développementale de l'Université de Bordeaux 2. 


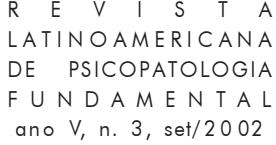

\section{Le dispositif de recherche}

Les entretiens semi-directifs ont été réalisés dans deux services différents du Centre Hospitalier Régional de Bordeaux. Dans les deux services ont été utilisés les mêmes principes pour rencontrer les patients, les mêmes critères de recrutement, le même entretien semi-directif et les mêmes principes de passation de ce dernier.

La rencontre du patient avec le psychologue intervient à la suite d'une consultation médicale. C'est le médecin qui le premier informe le patient au sujet de la recherche et sollicite sa participation. Si ce dernier accepte, il rencontre alors le psychologue qui expose les principes de la recherche de manière détaillée.

Les critères d'inclusion dans la recherche sont au nombre de deux:

- le patient consomme des médicaments psychotropes (anxiolytiques

- antidépresseurs et/ou hypnotique) depuis trois mois au moins

- la première prescription a été effectuée par un médecin généraliste

Les critères d'exclusion sont au nombre de deux:

- la prescription a été motivée par des troubles psychotiques (aucun des patients de notre échantillon ne recourt aux neuroleptiques)

- la prescription est en relation avec un alcoolisme passager ou avéré.

Trois thèmes sont explorés à l'aide de l'entretien semi-directif. Le premier concerne la consommation de médicaments psychotropes et il est introduit par la question: est-ce que vous pourriez me dire comment a commencé cette consommation de médicaments? Comme nous l'avons montré ailleurs (DucoussoLacaze et Keller, 2001) cette question supporte la mise en intrigue (Ricoeur, 1983) et donc la temporalisation de l'expérience subjective associée à la consommation de médicaments ainsi que de l'expérience de la souffrance psychique.

Le second thème vise plus directement la souffrance psychique elle-même et il est introduit par la question: Au fond, de quoi souffrez-vous?

Enfin le troisième thème est destiné à explorer la familiarité du patient avec "le psychologique". Il est introduit par la question: Est-ce que vous pensez que cette souffrance dont vous avez parlé pourrait être traitée autrement que par des médicaments psychotropes? Précisons que ce thème a été conçu pour permettre de solliciter la participation du patient à un suivi psychologique de deux ans à raison d'une rencontre tous les deux mois. Suivi psychologique qui, dans le cadre de notre plan d'expérience devait permettre d'appréhender l'évolution du discours des patients par rapport à la consommation de médicaments psychotropes.

Tous les entretiens semi-directifs ont été enregistrés sur une bande magnétique audio, avec l'accord des patients. Ils sont ensuite transcrits. A ce jour sur la quarantaine d'entretiens effectués, trente ont été intégralement transcris, lus et soumis à la grille d'interprétation que nous présentons ici. 


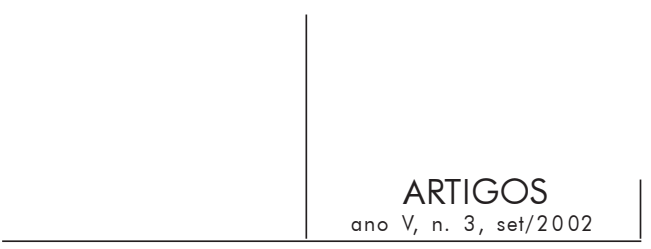

Soulignons un dernier point. L'analyse ne s'épuise pas dans la mise en forme des configurations narratives. Et cela tient directement au fait que le cadre de l'entretien a été conçu à partir d'une référence à la théorie psychanalytique. Sont déterminantes, en l'occurrence, toutes les relances destinées à explorer comment les patients pensent leur consommation de médicaments psychotropes en relation avec leur entourage et notamment leur entourage familial. Connaissent-ils des personnes qui consomment ces médicaments ou qui en ont consommé? Connaissent-ils des personnes ayant eu à faire à la souffrance psychique? Pour treize des patients rencontrés ces questions actualisent clairement des représentations liées à la filiation narcissique. Il est alors possible de caractériser la place que prennent la consommation de médicaments psychotropes, l'acte de prescription et les paroles prononcées par le médecin généraliste dans les fantasmes de la filiation narcissique.

\section{La filiation narcissique: bref rappel}

Cette notion, on le sait, prend tout son sens dans le cadre de la distinction proposée par Guyotat (1996) entre filiation instituée et filiation narcissique. Si la première situe le sujet par rapport à ses ascendants et descendants en fonction de règles sociales, la seconde relève de la vie fantasmatique du groupe et du sujet. Elle est en relation directe avec l'imaginaire et les désirs du sujet et de son groupe d'appartenance à propos d'une filiation "rêvée". Elle engage donc des fantasmes concernant une filiation idéale et, en cela, est en prise directe avec le narcissisme du groupe et du sujet.

On sait depuis Freud (1973) que les productions fantasmatiques à propos de son origine jouent un rôle de première importance dans la construction identitaire de tout être humain. Nous voulons parler bien sûr de la notion de roman familial, cet ensemble de fantaisies diurnes qui sont autant de variations autour de la scène primitive. Les travaux psychanalytiques sur la famille ont permis de comprendre que s'y ajoutent des productions fantasmatiques du groupe concernant la place imaginaire de chacun dans la famille et concernant l'origine du groupe familial. Ce qui a amené Legendre à estimer que "sous le cadre légal de référence est toujours à l'œuvre une généalogie occulte" (1990, p. 92). On peut alors parler de filiation narcissique groupale à partir, notamment, des notions de mythe familial et de fantasme de transmission. Le mythe familial est ce discours conscient que tient la famille à propos de son origine mais aussi à propos de ce qui, pour ses membres, relève de l'énigme (Eiguer, 1987). Le mythe familial a une fonction explicative en ceci qu'il dit comment ce qui fait problème a 


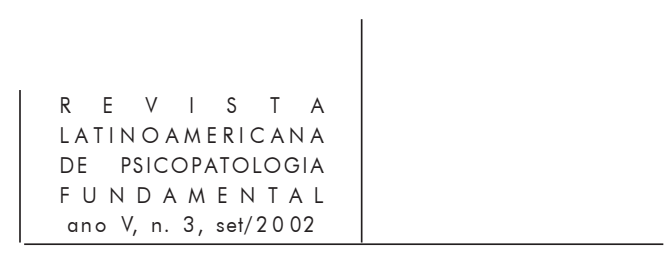

commencé. Ainsi, dans nos entretiens semi-directifs, les patients reprennent fréquemment à leur compte le mythe familial pour dire comment leur souffrance ou la souffrance familiale a commencé. Quant à la notion de fantasme de transmission (Ciccone, 1999), elle permet de rendre compte d'une position subjective qui amène les sujets à se situer, ou à situer un membre du groupe familial, consciemment ou inconsciemment, en tant qu'héritier d'un objet psychique familial: trait de caractère mais aussi angoisse ou anxiété comme nous le verrons dans les deux exemples ci-dessous.

\section{Entretien avec Madame S.}

Madame S. a trente deux ans, elle est mariée et a deux petites filles. Elle consulte dans le service de clinique du sommeil pour des insomnies persistantes. Pour la clarté de la présentation je retiendrai deux séquences narratives prélevées dans l'ensemble de son récit.

Première séquence narrative: une série de drames

Suite à la question d'ouverture de l'entretien (Est-ce que vous pouvez me dire comment ça a commencé cette consommation de médicaments?), Madame $S$. évoque une série d'événements très douloureux qui se sont succédés de manière surprenante. Alors qu'elle avait "une vingtaine d'années" son père a été hospitalisé "de force" en psychiatrie, sa mère a eu un cancer, sa sœur est tombée gravement malade. "J'ai soigné les trois en travaillant" dit-elle. Puis le concubin de sa sœur est mort d'un cancer et "c'est moi qui ai aidé ma sœur à surmonter ça". Elle précise alors: "En fait ça a été un choc émotionnel qui a duré trois ans". L'hospitalisation de son père lui laisse un souvenir extrêmement douloureux d'autant que, selon ses propres mots, "Il avait complètement disjoncté. Il ne voulait plus de moi. Il m'a dit des horreurs".

C'est dans le cadre de cette séquence narrative qu'intervient l'évocation du médecin prescripteur (médecin généraliste). Madame S. dit: "C'était normal que je pleure. Quand vous avez un père qui devient fou, qui vous dit des horreurs, une mère malade, crevée. Le docteur me l'a dit 'prenez un peu de Lexomil'”. Retenons ce point: la prescription de médicaments psychotropes et l'énoncé du médecin se situent sur cette configuration narrative de l'expérience d'événements traumatiques. Subjectivement ils semblent attester, pour la patiente, qu'elle a souffert et continue de souffrir de ce "choc émotionnel" non dépassé en quelque sorte. 


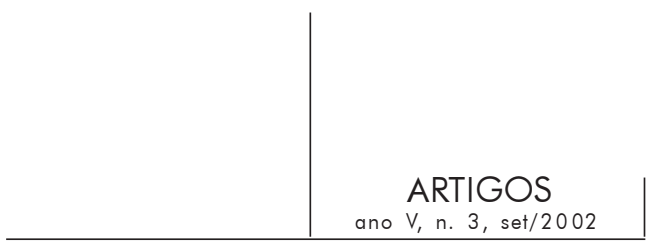

Deuxième séquence narrative: un trait de caractère hérité

Dans la suite de l'entretien la patiente évoque un trait de caractère: "J'étais une enfant un peu trop sensible". Ma relance, en forme d'interrogation, sur cette expression suscite un récit à propos de son enfance, "une enfance peureuse tristounette, très sérieuse, trop sérieuse. J'ai ce tempérament là déjà en moi". Mais, fait remarquable, la patiente reprend le discours de sa mère: "C'est maman qui me l'a dit". Elle en vient alors à dire qu'elle a hérité du caractère de son père: "Un homme très travailleur, très rigoriste". Est donc mobilisé un fantasme de transmission conscient très certainement soutenu par le mythe familial dans la mesure où la patiente précise que, si elle a hérité de son père, sa sœur en revanche a hérité de sa mère. On sait que le mythe familial dit souvent l'origine du caractère de chacun et l'inscrit dans une lignée spécifique. En vertu de ce mythe Mme. S serait donc comme son père qui, dit elle, "travaillait toujours, toujours, qui est d'une intelligence prodigieuse. Malheureusement j'ai hérité de lui, de son côté travailleur acharné, perfectionniste mais pas l'intelligence et la mémoire".

Fantasme de transmission qui l'amène donc à se situer en position d'héritière d'un objet psychique paternel. Mais un objet psychique autant aimé que haï. Et, à la fin de l'entretien elle me confie "Quand M. C. (Le médecin du service de clinique du sommeil) m'a proposé de rencontrer un psychologue, je me suis dit: c'est lui (son père) qui est fou, pas moi".

La rencontre avec le psychologue réactualise donc pour la patiente son identité d'héritière d'une transmission psychique qui, sur le plan imaginaire, menace de se réduire à la part haïe de l'héritage. Le traitement par les médicaments psychotropes, au travers de son ancrage subjectif dans les paroles du médecin généraliste, semble en revanche attester qu'elle souffre bien de traumatismes (ou chocs émotionnels), ce qui semble garantir une différenciation minimale à l'égard de l'héritage familial. Tout se passe comme si, sur le plan fantasmatique, il constituait une sorte de rempart contre les risques d'indifférenciation avec la figure paternelle. Un rempart toutefois extrêmement menacé par un désir d'appartenance et une forme de collage au discours familial.

Une remarque s'impose: ce collage au discours familial peut-il être mis en relation avec ce que Legendre appelle collage Oedipien? S'il en était ainsi cela signifierait que le désir de ressemblance au père dissimule un désir inconscient inacceptable. Hypothèse plausible mais difficilement vérifiable. Elle correspondrait pourtant à cette idée de Ciccone selon laquelle le fantasme de transmission a une double fonction, celle d'inscrire le sujet dans sa filiation et celle de le dédouaner à l'égard de ses désirs inconscients. Dans un tel cas de figure le traitement par les médicaments psychotropes aurait pour fonction subjective de maintenir en 


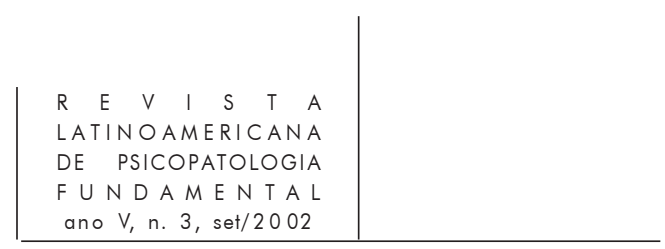

l'état l'attachement œdipien à la figure paternelle tout en donnant sur les plans conscient et préconscient le sentiment d'une différenciation possible.

\section{Entretien avec Madame X}

Elle aussi est rencontrée par le psychologue (P-H Keller) dans le service de "Clinique du Sommeil". L'entretien fait apparaître qu'elle s'est mariée il y a un peu moins d'un an. Les situations professionnelles de chacun des membres du couple ont fait qu'ils n'ont pas vraiment vécu ensemble depuis qu'ils se connaissent. D'autre part elle a une demi-sœur d'un premier mariage de sa mère. Celle-ci a quatorze ans de plus qu'elle, il en est beaucoup question dans l'entretien.

Dès le début de l'entretien elle dit des médicaments psychotropes, "Moi j'en pense en partie du bien, puisque j'ai été confrontée à certains problèmes et que grâce à eux j'ai pu retrouver le sommeil (...) mais mon problème, c'est un problème de dépendance!, Ca a été ma grande... le grand problème de mes médecins, ma grande préoccupation par la suite... donc il faut faire attention et là en ce moment je souhaite absolument diminuer les doses et arriver à m'en passer tout à fait”".

Retenons trois éléments parmi ces premières paroles.

1. Le mot de dépendance qui qualifie sa relation aux médicaments.

2. L'hésitation de sa parole associe d'emblée la dépendance aux paroles des médecins. Ainsi il est difficile de savoir si c'est son problème ou celui des médecins.

3. La consommation de médicaments est étroitement associée à l'idée d'un danger pour le sujet.

Comme pour la présentation précédente je retiendrai des séquences narratives, trois en l'occurrence.

\section{Première séquence narrative: la pharmacie familiale}

La question à propos de l'existence d'éventuels consommateurs de médicaments dans son entourage conduit Mme. $\mathrm{X}$ à recourir au mythe familial de sa famille d'origine. C'est en fait au discours de ses parents à propos de leur propre consommation qu'elle fait appel. Le père et la mère en ont consommé et en consomment encore. Voilà pour les souvenirs qui appartiennent en propre à la patiente. Le mythe familial, lui, propose une explication à propos de cette consommation, il dit comment elle a commencé. 


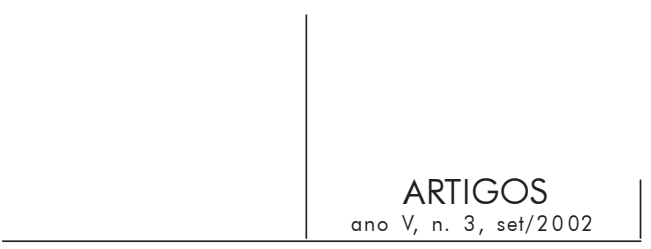

Selon ce mythe, son père a commencé à consommer des médicaments psychotropes à la suite des événements qu'il a vécus pendant la guerre d'Algérie. Et la patiente ajoute "Il a été dépendant pendant de nombreuses années". Remarquons que le signifiant "dépendant" qualifie la relation du père aux médicaments, de même qu'il a qualifié la sienne au début de l'entretien. Ce signifiant était-il employé dans sa famille? Quel danger évoquait-il pour la petite fille? Difficile de répondre à ces questions. Pourtant dans la continuité de cette mobilisation du mythe familial la patiente en vient à se situer en tant qu'héritière et sa parole laisse affleurer son ambivalence à l'égard de cet héritage "Oui, d'ailleurs je lui disais qu'il arrête. Oui, et puis finalement je reproduis le même schéma". La mobilisation du mythe familial ici encore supporte la création d'un fantasme de transmission conscient, teinté de déception narcissique. Ce fantasme de transmission va s'étendre à la mère de la patiente.

Le mythe familial raconte aussi que la mère de la patiente a commencé de consommer des médicaments psychotropes après la naissance de la patiente. Mais ce ne serait pas la naissance qui en est la cause, plutôt l'installation à la maison de la grand-mère paternelle avec laquelle la mère ne s'entendait pas du tout. La patiente dit alors "Oui, elle a un tempérament stressé et anxieux de nature, elle me l'a passé c'est sûr. Le fait de vivre avec des personnes constamment angoissées, forcément ça déteint un peu”. Comme on le voit, c'est à nouveau un fantasme de transmission qui est à l'œuvre. Mme. X se situe en tant qu'héritière d'un objet psychique parental: l'anxiété, l'angoisse. D'ailleurs elle en vient à évoquer la "pharmacie familiale” qui, pour elle, témoigne de cette prégnance de l'anxiété chez ses parents. Sa mère ne cessait de lui donner des médicaments et elle-même, aujourd'hui encore, vit avec une "véritable pharmacie".

Deuxième séquence narrative: la demi-sœur et le beau-frère

Mais les habitudes de ses parents ne sont pas celles de sa demi-sœur qui, elle, avec son mari, refuse de consommer tant de médicaments. Sa demi-sœur a d'ailleurs été "épouvantée, elle qui était sortie un peu plus tôt de ce milieu familial”, lorsqu'elle a vu tous les médicaments que consomme madame X. Mais à propos de sa demi-sœur et de son beau-frère Mme. X donne des précisions intéressantes. Cette demi-sœur, à qui elle souhaitait rendre visite avant de se "retrouver seule avec son mari", occupe pour elle une place imaginaire tout à fait particulière "je suis un peu sa première fille” dit Mme. X en rappelant que celle-ci est son aînée de 14 ans. De plus elle précise que son beau-frère, alors qu'elle était triste de le voir lui enlever sa demi-sœur, lui a dit: "Tu sais tu seras toujours un petit peu notre fille". 


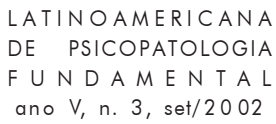

Nous pouvons préciser l'idéal familial dont est porteuse Madame X. Au couple parental institué transmetteur d'angoisse s'oppose polairement le couple idéalisé de la sœur et du beau-frère. Au plan préconscient la consommation de médicaments psychotropes la désigne en tant qu'héritière de son père et de sa mère et réactualise la difficulté de s'aimer soi-même en tant qu'enfant de ce couple là dont le couple idéalisé constitué par la sœur et le beau-frère révèle les failles. Les médicaments, avec l'énoncé des médecins qui accompagne leur consommation, sont donc au cœur d'un conflit entre deux places fantasmatiques incompatibles.

\section{Troisième séquence narrative: la solitude des femmes de la famille}

Lorsque le psychologue interroge le sentiment de solitude qu'elle dit éprouver dans la région où elle est obligée de vivre pour des raisons professionnelles, le mythe familial est à nouveau sollicité. Selon celui-ci, la mère et la demi-sœur ont également éprouvé un tel sentiment lorsqu'elles se sont mariées. Elles aussi ont dû quitter leur région d'origine, pour suivre leur mari (La mère et le père se sont installés dans la même région que madame $\mathrm{X}$. après son mariage). Ainsi selon ce mythe familial le mariage se présente comme une épreuve pour les femmes de la famille. D'ailleurs dès le début de l'entretien madame X. a dit que l'angoisse et l'insomnie ont surgi au moment de son mariage: elle ne voulait "pas rester à $Y$. (région d'adoption) toute seule avec (s)on mari". Or, si la mère a beaucoup souffert de cette solitude, la demi-sœur en revanche l'a rapidement surmontée. Et lorsque le psychologue demande à madame X. si elle en a parlé avec cette dernière, les places de la filiation instituée dans le discours ne sont plus du tout assurées: "pas vraiment parce qu'on a quand même 14 ans d'écart. C'est un peu ma seconde maman plutôt que ma petite sœur. Ma sœur a vécu avec nous jusqu'à ce que j'ai 8 ans et c'était vraiment la seconde fille. Enfin j'étais le seconde fille et c'est la fille aînée et donc vraiment on a eu des rapports... très privilégiés. J'étais sa... sa première fille en quelque sorte”.

Qui est la mère de madame X est-on tenté de demander? Sa mère ou sa demi-sœur? Mais on peut aller plus loin. De qui faut-il être la fille pour pouvoir faire sa vie avec un homme? Etre la fille de sa mère, ce serait reconduire l'épreuve de celle-ci et enfanter dans les mêmes conditions qu'elle (Madame X. confie qu'elle vient de dire à son médecin qu'elle souhaite avoir un enfant), être à sa place probablement. Ainsi les fantasmes de la filiation narcissique semblent dissimuler un conflit inconscient en rapport avec la sexualité et lié à une forme de collage aux images maternelles. Pour Madame X., la consommation de médicaments psychotropes et le discours médical à leur sujet entérinent les données de ce conflit inconscient. 


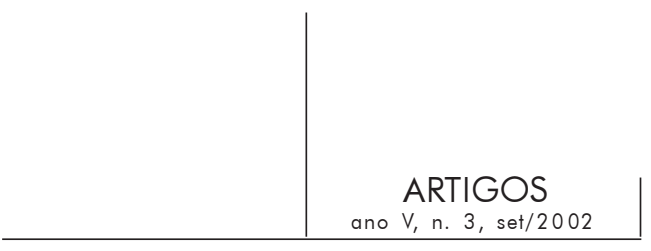

Conclusion

Adoptant une perspective anthropologique Guyotat propose l'hypothèse suivante: "Dans notre culture scientifique médicale, c'est le corps malade qui a pris la place des esprits. C'est lui le persécuteur et c'est sur lui que l'on doit agir par des traitements qui lui sont destinés, éventuellement par des médicaments psychotropes" (1996, p. 50).

Je reformulerai cette hypothèse avec mon propre vocabulaire tout en tentant de l'articuler avec les idées que j'ai avancées sur la base de nos données cliniques.

Du point de vue de la subjectivité du patient, la prescription de médicaments psychotropes atteste qu'il y a de "l'autre en soi", qu'est à l'œuvre une altérité qui affecte et détermine le sujet à son insu. En cela les médicaments psychotropes participent, dans notre culture laïque et scientifique, au processus de recomposition de l'altérité (Ducousso-Lacaze, 2001). Mais le dispositif de prescription dans sa dimension technique n'atteste pas seulement de cette altérité, il en propose une représentation: le corps, le système nerveux.

Nos entretiens mettent en évidence que c'est sur cette représentation de l'altérité que les fantasmes des patients et la logique du dispositif divergent. Par l'intermédiaire des fantasmes de la filiation narcissique, un certain nombre de nos patients élaborent leur propre représentation de ce qui les détermine, de ce qui les fait souffrir. Cette représentation de l'altérité revêt alors dans certains cas la figure de l'ancêtre (le grand-père ou la grand-mère parfois), la mère, le père. Et ceci en fonction des enjeux narcissiques d'appartenance et de différenciation à l'égard du groupe familial et des énoncés identificatoires dont il est porteur. Mais aussi en fonction d'une double contrainte: l'exigence de satisfaction des désirs inconscients et l'exigence de n'en rien savoir.

Les propos tenus par le médecin au moment de la prescription revêtent alors une signification toute particulière et, pour une grande part, indépendante du dispositif rationnel. Les deux exemples que nous avons retenus montrent qu'ils peuvent eux-mêmes acquérir le statut d'énoncés identificatoires et, sur le plan fantasmatique, soutenir l'opération subjective de transformation des médicaments psychotropes soit en support de la transmission psychique, soit en instrument de lutte contre cette transmission. Ce qui probablement ouvre la voie à une meilleure compréhension des conduites dites de non observance (refus de recourir à ces médicaments, automédication, "prescription" à des proches). 


\section{References}

Ciccone, A. La transmission psychique inconsciente. Paris: Dunod, 1999.

Ducousso-Lacaze, A. Nos psychothérapies sont-elles modernes? L'évolution psychiatrique, 66, 516-26, 2001.

Ducousso-Lacaze, A. et Keller, P-H. Récit de l'expérience de consommation de médicaments psychotropes et récit de soi. Cliniques Méditerranéennes, 65, 291305, 2001.

Ehrenberg, A. L'individu incertain. Paris: Calman-Lévy, 1995.

EIGUER, A. La parenté fantasmatique. Paris: Dunod, 1987.

Freud, S. Le roman familial des névrosés. In: Résultats, idées, problèmes - II. Paris: PUF, 1973.

GuYotat, J. Filiation et puerpéralité. Paris: PUF, 1996.

Le Moigne, P. Anxiolytiques, hypnotiques. Les facteurs sociaux de la consommation. Documents du Groupe de Recherche Psychotropes Politique et Société, n. 1, 1999. Legendre, P. Filiation. Paris: Fayard, 1990.

Ricceur, P. Temps et récit. Paris: Seuil, 1983.

ZARIFIAN, E. Mission générale concernant la prescription et la consommation de médicaments psychotropes en France. Rapport établi à la demande du ministre délégué à la santé, 1996.

\section{Resumen}

A partir de duas entrevistas semi-diretivas o autor apresenta alguns aspectos dos resultados de uma pesquisa clínica realizada com pacientes do hospital geral consumindo remédios psicotrópicos. Trata-se de evidenciar como, para alguns pacientes, o consumo e a prescrição tomam um sentido relacionado às questões em jogo na filiação narcisica. As análises clínicas remetem essencialmente à função de suporte imaginário de transmissão que os remédios podem revestir, tanto quanto ao seu papel nos conflitos entre pertinência e diferenciação em relação ao grupo familiar.

Palavras-chave: Filiação narcisica, remédios psicotrópicos, fantasma de transmissão, pertinência, diferenciação

Partiendo de dos entrevistas semi dirigidas el autor presenta algunos aspectos de los resultados de una investigación clínica realizada con pacientes de un hospital general medicados con psicotrópicos. Se trata de poner de relieve cómo, para ciertos pacientes, el consumo y la prescripción toman un sentido en el marco de la filiación narcisista. Los análisis clínicos insisten sobre la función de soporte imaginario de la 


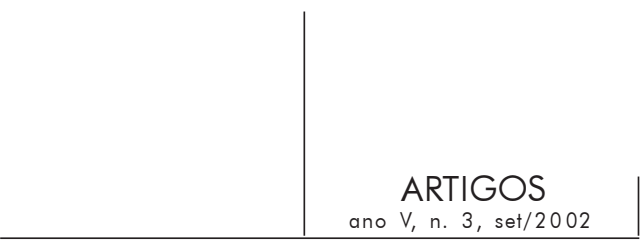

transmisión que puede revestir a los medicamentos así como sobre su rol en los conflictos entre pertenencia y diferenciación con respecto al grupo familiar.

Palabras llave: Filiación narcisista, medicamentos psicotrópicos, fantasía de transmisión, pertenencia diferenciación

Based on two semi-directed interviews, the author presents aspects of a clinical investigation carried out with patients of a general hospital who consume psychoactive medications. The author stresses that, for certain patients, consumption and prescription take on a meaning related to narcissistic filiation. The clinical analyses indicate the possible function of the imaginary support from the transmission of medications, as well as on their role in conflicts between belonging and differentiation related to the family group.

Key words: Narcissistic filiation, psychoactive medication, fantasy of transmission, belonging, differentiation

Versão inicial recebida em março de 2002

Versão revisada recebida em agosto de 2002 